\title{
A new consensus document on electrocardiographic interpretation in athletes: does it help to prevent sudden cardiac death in athletes?
}

\author{
N. M. Panhuyzen-Goedkoop ${ }^{1,2} \cdot$ H. T. Jørstad' J. L. R. M. Smeets ${ }^{2}$ \\ Published online: 1 February 2018 \\ (c) The Author(s) 2018. This article is an open access publication.
}

\begin{abstract}
Sudden cardiac arrest or death (SCA/SCD) in athletes has a low event rate. Pre-participation or eligibility screening is a widely accepted method of primary prevention of SCA/SCD in athletes. Most European countries and international sports governing bodies perform ECG-inclusive screening. However, implementation of a resting 12-lead ECG in pre-participation or eligibility cardiac screening is still a topic of debate. Recently, the 'International recommendations for electrocardiographic interpretation in athletes' was published in three leading international medical journals. These international ECG criteria are based on studies with detailed information on resting 12-lead ECG of Caucasian and Afro-Caribbean athletes or on consensus in case evidence was lacking. Normal, borderline and abnormal ECG findings in young athletes (age 12-35 years) are clearly described and illustrated to assist the screening physician in interpreting ECGs of athletes correctly.

In this 'point of view paper' we will discuss whether these new ECG criteria actually help prevent SCA/SCD in athletes.
\end{abstract}

Keywords Athlete $\cdot$ ECG $\cdot$ Sudden cardiac death $\cdot$ Pre-participation screening $\cdot$ Prevention

\section{Introduction}

Sudden cardiac arrest and/or death (SCA/SCD) in athletes is a very tragic event that attracts a lot of media attention. The key question conventionally raised after such an event resulting from lethal ventricular arrhythmia, i. e. ventricular tachycardia/fibrillation (VT/VF), is: could this have been prevented? SCD in competitive athletes aged 35 years and younger (young athletes) is rare (0.6-2.85/100,000 annually). The incidence is considerably lower than in the overall population (3-10.7/100,000 annually) and significantly lower than the incidence of VT/VF in Europe (84.0/100,000 annually) [1-7]. Most inherited and congenital cardiovascular diseases (CVD) in athletes at risk of VT/VF can be identified during life $[2,3]$. To date, primary prevention with pre-participation or eligibility cardiac screening is a widely accepted method to reduce SCA/SCD in athletes [8-10]. If pre-participation or eligibility cardiac screening

N. M. Panhuyzen-Goedkoop

n.m.panhuyzen@amc.nl

1 Academical Medical Centre Amsterdam, Amsterdam, The Netherlands

2 Radboud University Medical Centre, Radboudumc, Nijmegen, The Netherlands fails to identify athletes at risk, secondary prevention with bystander resuscitation, including defibrillation with automatic external defibrillator (AED), is essential to save an athlete's life [11]. However, an AED is no adequate replacement for pre-participation or eligibility cardiac screening [11]. How we need to screen athletes for conditions predisposing to VT/VF optimally is a topic of debate.

In this manuscript we discuss the 'International recommendations for electrocardiographic interpretation in athletes', questioning if these new ECG criteria help preventing SCA/SCD in athletes [12-14].

\section{Pre-participation cardiac screening}

The purpose of pre-participation or eligibility cardiac screening in athletes is identifying CVD at risk of VT/VF and reducing SCA/SCD by disease management [8-10]. Pre-participation or eligibility cardiac screening consists of personal and family history taking and physical examination $[1,8-10,15]$. Pre-participation or eligibility cardiac screening performed by most European countries and international sports governing bodies include a 12-lead resting ECG $[1,8,16]$. In the Netherlands, pre-participation or eligibility cardiac screening is performed by sports physicians according to the 'Lausanne protocol' [15]. If pre- 
Table 1 Comparison of three different sets of ECG criteria in ECG-inclusive screening in three cohorts of young athletes

\begin{tabular}{|c|c|c|c|c|c|c|c|}
\hline \multirow[b]{2}{*}{ Reference } & \multirow[b]{2}{*}{ Cohort } & \multicolumn{2}{|c|}{ ESC 2010 criteria } & \multicolumn{2}{|c|}{ Seattle criteria } & \multicolumn{2}{|c|}{ Refined criteria } \\
\hline & & Caucasian & $\begin{array}{l}\text { Afro/ } \\
\text { Caribbean }\end{array}$ & Caucasian & $\begin{array}{l}\text { Afro/ } \\
\text { Caribbean }\end{array}$ & Caucasian & $\begin{array}{l}\text { Afro/ } \\
\text { Caribbean }\end{array}$ \\
\hline & & FPR $(\%)$ & FPR (\%) & $\operatorname{FPR}(\%)$ & FPR $(\%)$ & $\operatorname{FPR}(\%)$ & FPR $(\%)$ \\
\hline Sheikh [20] & $\begin{array}{l}\text { Caucasian 4,297, } \\
\text { Afro/Caribbean } \\
\text { 1,208; male } 94.2 \% \text {; } \\
\text { 2000-2012 }\end{array}$ & 26.5 & 59.9 & 7.9 & 20.7 & 6.1 & 15.8 \\
\hline Riding [21] & $\begin{array}{l}\text { Caucasian 367, Afro/ } \\
\text { Caribbean 748; male } \\
\text { 100\%; 2010-2014 }\end{array}$ & 12.6 & 15.5 & 3.9 & 5.3 & 2.5 & 3.1 \\
\hline Malhotra [22] & $\begin{array}{l}\text { Caucasian 9,262, } \\
\text { Afro-Caribbean } 894 \text {; } \\
\text { adolescent soccer } \\
\text { players }\end{array}$ & 18.6 & 29.1 & 8.5 & 15.8 & 2.1 & 9.2 \\
\hline
\end{tabular}

ESC European Society of Cardiology, FPR false positive rate

Table 2 International ECG criteria: normal and borderline ECG findings in athletes 35 years and younger [12-14]

\begin{tabular}{lll}
\hline Cardiac adaptation & Normal ECG finding & Borderline ECG finding \\
\hline Vagotonia & Sinus bradycardia or arrhythmia & \\
& Ectopic atrial or junctional rhythm & \\
& 1st degree AV block & \\
& Wenckebach AV block & Complete RBBB \\
Changes of cavity size and wall & Incomplete RBBB & Left atrial enlargement \\
thickness (cardiac remodelling) & & Right atrial enlargement \\
& Increased QRS voltage for LVH or RVH & Left axis deviation \\
& Early repolarisation/ST-segment elevation & Right axis deviation \\
& ST-segment elevation followed by T-wave inversion leads V1-4 in & Afro-Caribbean athletes \\
& T-wave inversion leads V1-3 age 16 years and younger & \\
\hline
\end{tabular}

$A V$ atrioventricular, $E C G$ electrocardiogram, $R B B B$ right bundle branch block, $L V H$ left ventricular hypertrophy, $R V H$ right ventricular hypertrophy

participation or eligibility cardiac screening results raise suspicion of a CVD at risk of VT/VF additional cardiac evaluation is recommended before clearing the athlete $[8,9$, 17]. To adequately perform pre-participation or eligibility cardiac screening, the screening physician needs training and skills in physiology, ECG interpretation, CVD at risk of VT/VF, and CVD management in athletes [10, 18].

\section{Athlete ECG}

In 2010, the European Society of Cardiology (ESC) for the first time classified athlete ECGs in training-related and training-unrelated or pathologic ECG findings [16]. Training-related ECG findings induced by vagotonia and volume and/or pressure overload of the cardiac cavities are an expression of athlete's physiologic cardiac adaptation or remodelling [16, 19, 20]. The ESC 2010 criteria were principally based on a large Italian registry in almost exclusive Caucasian young athletes $[2,16]$. Left atrial enlarge- ment, cardiac axis deviation and criteria of right ventricular hypertrophy (RVH) were classified as pathologic ECG changes [2, 16]. However, the typical ECG changes of left ventricular hypertrophy (LVH) and ST-T changes in AfroCaribbean ethnicity were not mentioned and therefore regarded as abnormal. This resulted in a high false positive rate of ECG-inclusive screening (FPR 8.8-26.5\%) [20-22].

The Seattle 2013 criteria were based on ECG data in both Caucasian and Afro-Caribbean athletes [23, 24]. 'Convex ST-segment elevation combined with T-wave inversion in leads V1-4 in Afro-Caribbean athletes' was classified as training-related [24]. However, left atrial enlargement, cardiac axis deviation and RVH were still classified as abnormal [25].

The so-called 'borderline ECG findings' were introduced by Sheikh et al. because the issue of the resemblance of an athlete's ECG and pathology had not been solved [20]. The Refined criteria regarded left atrial and right atrial enlargement, cardiac axis deviation, RVH and T-wave inversion in leads V1-4 in Afro-Caribbean athletes nor- 


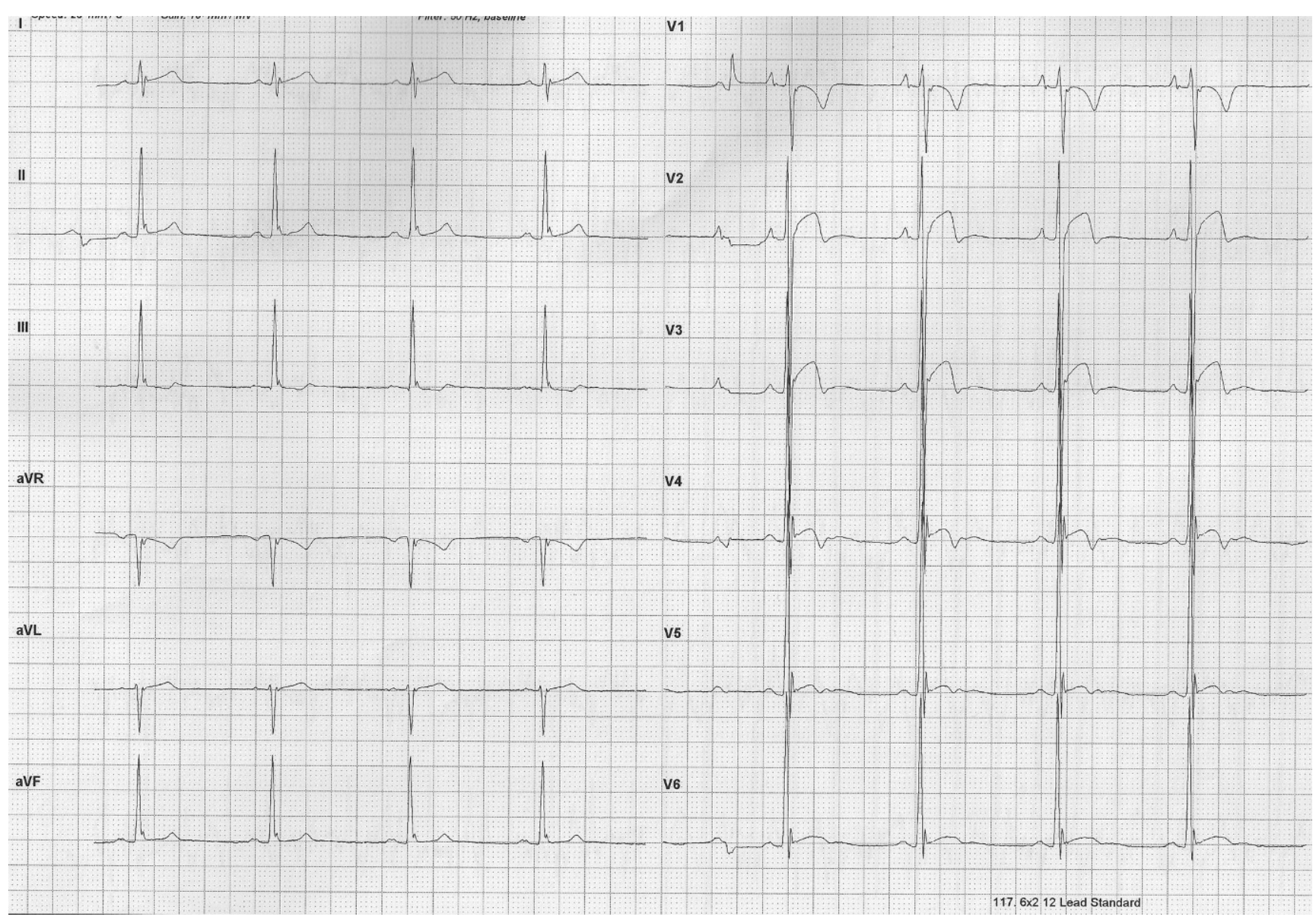

Fig. 1 ECG of an asymptomatic 21-year-old male Afro-Caribbean elite soccer player with a negative family history. Legend explanation: PR-interval $<120 \mathrm{~ms}$ and $<2.5 \mathrm{mV}$; J-elevation with convex ST-elevation and T-wave inversion in leads V2-4; increased QRS voltage criteria for LVH. In conclusion: normal ECG. Eligible to play. LVH left ventricular hypertrophy, ECG electrocardiogram. (Copyright 1999-2001 Jaeger bv)

mal findings if considered in isolation, but abnormal if two or more patterns were present [20]. Using the Refined criteria, a lower FPR was observed in a cohort of predominantly young $(\leq 35$ years) male Caucasian (FPR $6.1 \%$ ) and Afro-Caribbean (FPR 15.8\%) athletes (Table 1; [20]). A lower FPR using the Refined criteria was also observed in an Arabic study in young male athletes (FPR Caucasians 2.5\%, Afro-Caribbeans 3.1\%) and in adolescent soccer players (FPR Caucasians $2.1 \%$, AfroCaribbeans 9.2\%) (Table 1; [21, 22]). Although ECG screening demonstrated different FPR results in non-comparable cohort studies the Refined criteria were a major step forward in evidence-based ECG interpretation in athletes.

Research, in particular that of Sharma et al. (London, United Kingdom), conducted to describe in detail ECGs of athletes of different ethnicity, gender and intensity of sports participation has been of great value for the consensus of the international ECG criteria [12-14, 19, 20, 26, 27]. This third document, endorsed by several international (European Society of Cardiology, American Heart Association, American College of Cardiology) and national cardiac soci- eties, sports medicine societies and sports governing bodies, is an updated practical consensus for the screening physician to interpret and recognise physiologic and pathologic ECG findings in athletes [12-14].

\section{Normal, borderline and abnormal ECG findings in athletes}

The international consensus ECG criteria describe and illustrate each separate ECG finding at rest in young athletes [12-14]. The ECG findings are classified as normal, borderline and abnormal.

Normal or training-related ECG findings are induced by long-term sports participation on a regular basis for at least four hours per week [12-14]. As is mentioned above, these ECG findings at rest reflect increased vagotonia and cardiac remodelling in athletes (Table 2; Fig. 1). Such ECG findings warrant no further cardiac evaluation and the athlete is eligible to play. 


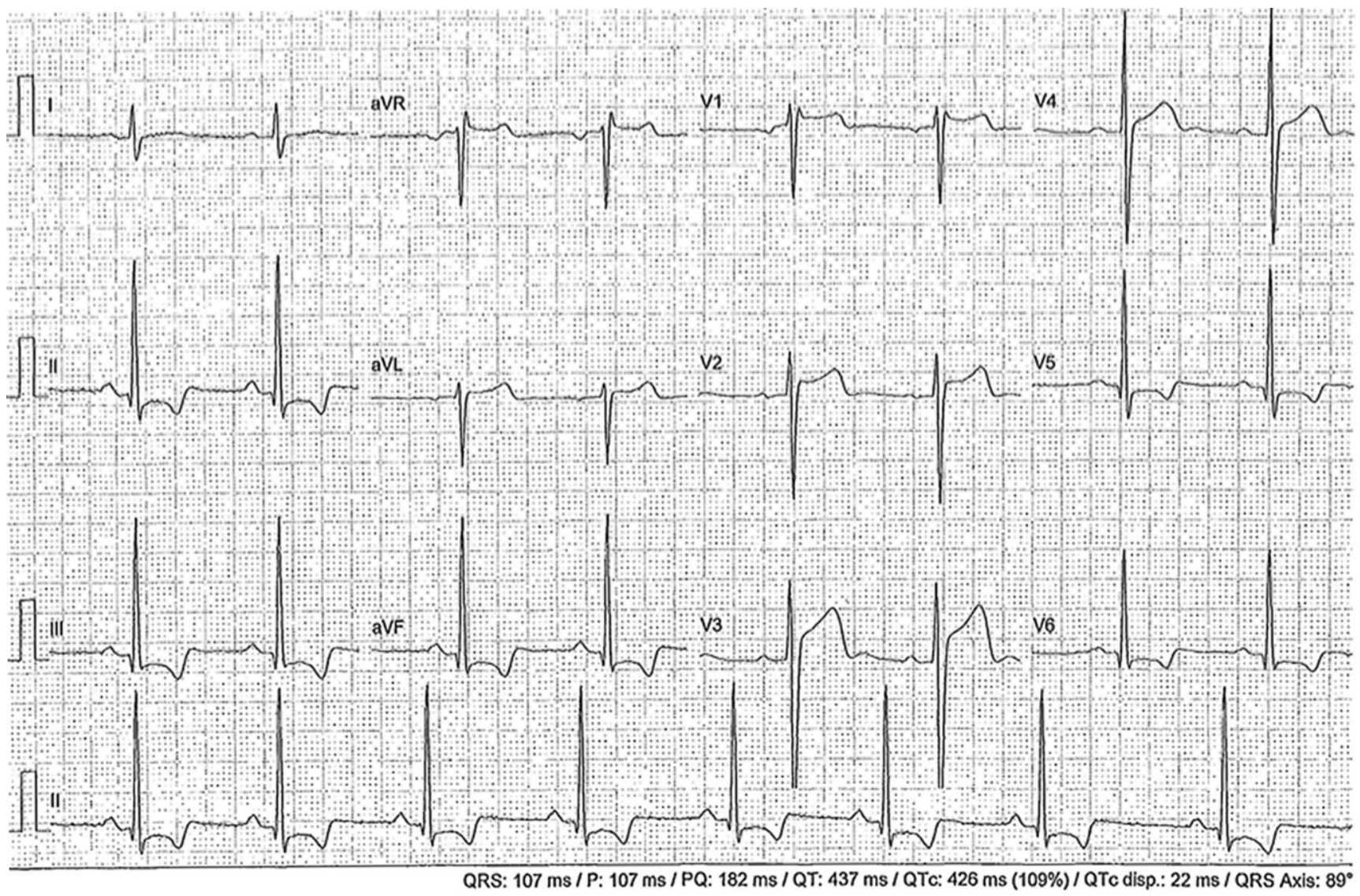

Fig. 2 Asymptomatic 23-year-old male Caucasian elite soccer player, negative family history, 5 years ago viral myocarditis. Legend explanation: PR interval $<120 \mathrm{~ms}$ and $<2.5 \mathrm{mV}$ (normal finding); ST-depression with negative T-waves in leads II-III-aVF, V5-6, i.e. inferolateral (abnormal finding). In conclusion: abnormal ECG. Perform additional cardiac evaluation with echocardiography and magnetic resonance imaging to rule out cardiomyopathy, hypertrophic or otherwise. Not eligible to play until further notice

Borderline ECG findings are left atrial and right atrial enlargement, electrical axis deviation and complete right bundle branch block (RBBB). When found in isolation in asymptomatic athletes with a negative family history of inherited CVD or SCA/SCD these findings are classified as normal and need no further cardiac evaluation (Table 2; [12-14]). However, when two or more borderline findings are present and/or the athlete is symptomatic and/or has a positive family history, these borderline ECG changes are classified as abnormal and additional cardiac evaluation is warranted before clearing the athlete [12-14].

Abnormal ECG findings are pathologic changes until proven otherwise. These abnormal findings reflect CVD at risk of VT/VF (i.e. inherited or congenital CVD, myocarditis, coronary disease) recommending additional cardiac evaluation before clearing the athlete to participate in sports (Figs. 2 and 3). The international ECG criteria clearly describe and illustrate the details of abnormal ECG findings suspicious of CVD at risk of VT/VF [12-14].

\section{Does this international consensus document help to prevent VT/VF in athletes?}

The consensus-based recommendations for ECG interpretation in athletes assist the screening physician in identifying athletes at risk of VT/VF. However, false positive rate (FPR) and false negative rate (FNR) of pre-participation or eligibility cardiac screening remain a problem. On the one hand, false positive test results wrongly identify athletes at risk of VT/VF, lead to unnecessary additional cardiac evaluation at high costs and result in uncertainty about the athlete's career $[27,28]$. On the other hand, wrong eligibility decision-making in false negative test results can put athletes at risk of VT/VF $[28,29]$. To reduce FPR results, the screening physician must be well-trained in interpreting ECGs of athletes and is recommended to use the most recent ECG criteria describing the lowest FPR [18, 20-22, 27, 30]. Future studies need to demonstrate that the international ECG criteria will result in a lower or equal FPR as compared with the Refined ECG criteria. However, there are several limitations inherent to ECG-inclusive pre-participation or eligibility cardiac screening, including the interpretation of an ECG at rest 


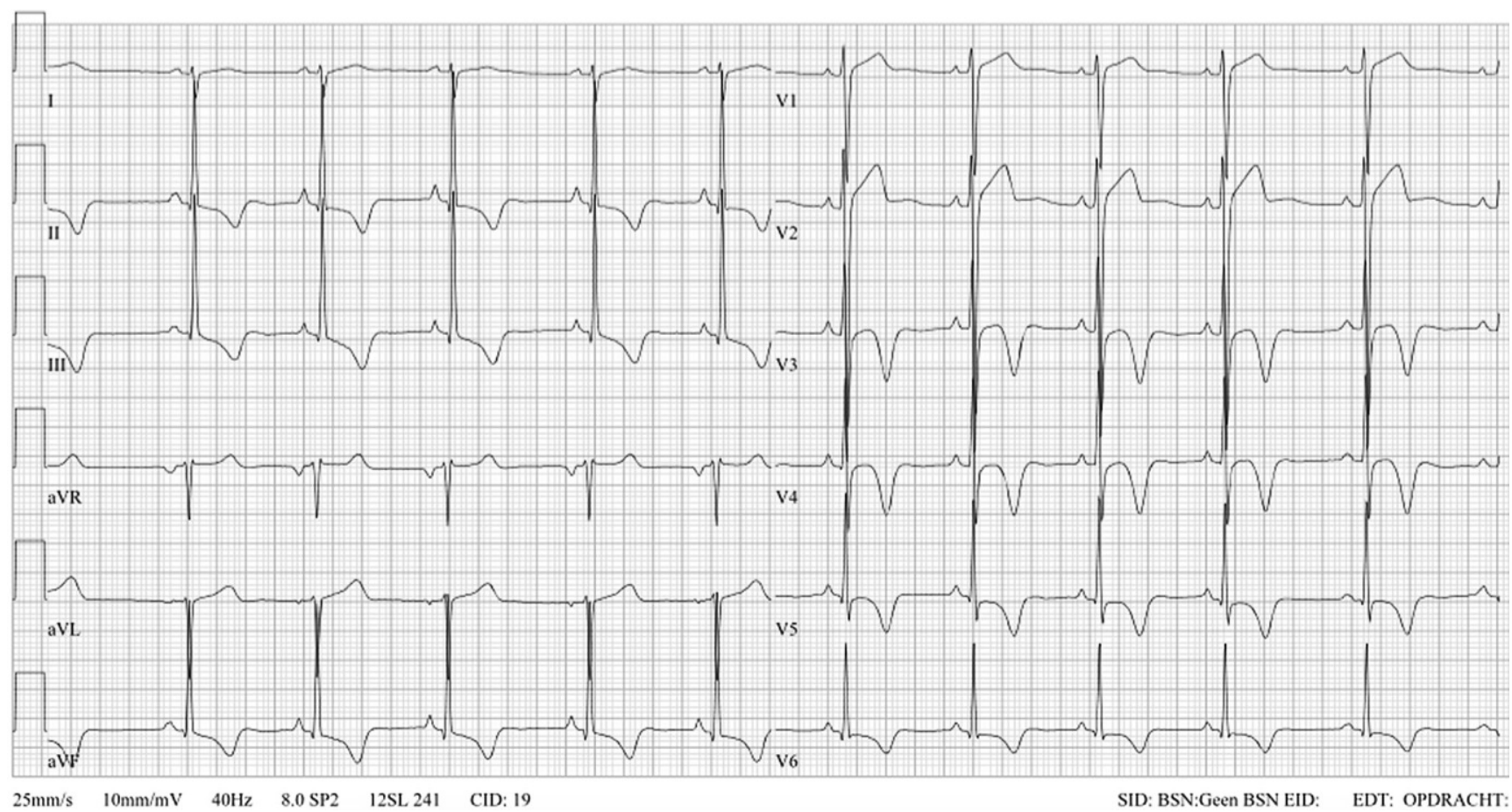

Fig. 3 Asymptomatic 21-year-old male Caucasian elite soccer player, on army check-up his 26-year old brother was referred for cardiac evaluation because of an abnormal ECG. Legend explanation: PR interval $<120 \mathrm{~ms}$ and $<2.5 \mathrm{mV}$ (normal finding); increased QRS voltage criteria for LVH (normal finding); ST-depression with deep negative T-waves in leads II-III-aVF, V3-6, i. e. infero-antero-lateral (abnormal finding). In conclusion: abnormal ECG. Perform additional cardiac evaluation with echocardiography and MRI to rule out cardiomyopathy. Not eligible to play until further notice. $L V H$ left ventricular hypertrophy, $E C G$ electrocardiogram, MRI magnetic resonance imaging

alone. Sometimes the ECG changes are very subtle and difficult to recognise. In myocarditis and cardiac concussion in blunt chest trauma these subtle ST-T changes are not always initially present. Furthermore, certain congenital and inherited CVDs (i.e. coronary anomaly, premature coronary syndrome, catecholaminergic polymorphic ventricular tachycardia) cannot be identified at a resting-ECG alone. Besides, abnormal ECG findings of a CVD at low risk of potential lethal cardiac events (i.e. atrial fibrillation, AV nodal re-entry tachycardia) are a confounder of the purpose of pre-participation or eligibility cardiac screening to identify athletes at risk $[1,4,5,10]$. Finally, ECG findings in other ethnicities, such as Hispanic, Asian and mixed ethnicity, and in athletes over 35 years of age and children are not included in this consensus document.

\section{Conclusion}

ECG-inclusive pre-participation cardiac screening to prevent SCA/SCD in athletes is implemented by most European countries and international sports governing bodies. The international ECG criteria, endorsed by international cardiac and sports medical societies and sports governing bodies, pose an updated and clear guide in interpreting ECGs of athletes to appropriately identify abnormal ECG findings at rest suspicious of CVD at risk of VT/VF. The screening physician must be trained in interpreting ECGs of athletes following the most recent ECG criteria to avoid wrong decision-making. Further studies are needed to determine if these updated international ECG criteria help to prevent SCA/SCD in athletes (12-35 years of age).

Conflict of interest N.M. Panhuyzen-Goedkoop, H.T. Jørstad and J.L.R.M. Smeets declare that they have no competing interests.

Open Access This article is distributed under the terms of the Creative Commons Attribution 4.0 International License (http:// creativecommons.org/licenses/by/4.0/), which permits unrestricted use, distribution, and reproduction in any medium, provided you give appropriate credit to the original author(s) and the source, provide a link to the Creative Commons license, and indicate if changes were made.

\section{References}

1. Bille K, Figuerias D, Schamasch P, et al. Sudden cardiac death in athletes: the Lausanne recommendations. Eur J Cardiovasc Prev Rehabil. 2006;13:859-75.

2. Corrado D, Basso C, Pavei A, et al. Trends in sudden cardiovascular death in young competitive athletes after implementation of a pre-participation screening program. J Am Med Assoc. 2006;296:1593-601.

3. Maron BJ, Doerer JJ, Haas TS, Tierney DM, Mueller FO. Sudden deaths in young competitive athletes: analysis of 1866 deaths in the United States, 1980-2006. Circulation. 2009;119:1085-92. 
4. Baggish AL, Hutter AM Jr, Wang F, et al. Cardiovascular screening in college athletes with and without electrocardiography: a crosssectional study. Ann Intern Med. 2010;152:269.

5. Harmon KG, Asif IM, Maleszewski JJ, et al. Incidence, etiology, and comparative frequency of sudden cardiac death in NCAA athletes: a decade in review. Circulation. 2015;132:10-9.

6. Risgaard B. Sudden cardiac death: a nationwide cohort study among the young. Dan Med J. 2016;B5321:63.

7. Berdowski J, Berg RA, Tijssen JG, Koster RW. Global incidences of out-of-hospital cardiac arrest and survival rates: systematic review of 67 prospective studies. Resuscitation. 2010;81:1479-87.

8. Corrado D, Pelliccia A, Bjornstad HH, et al. Cardiovascular preparticipation screening of young competitive athletes for prevention of sudden death: proposal for a common European protocol. Consensus Statement of the Study Group of Sport Cardiology of the working Group of Cardiac Rehabilitation and Exercise Physiology and the Working Group of Myocardial and Pericardial Diseases of the European Society of Cardiology. Eur Heart J. 2005;26:516-24.

9. Maron BJ, Levine BD, Washington RL, et al. Eligibility and disqualification recommendations for competitive athletes with cardiovascular abnormalities: task force 2: pre-participation screening for cardiovascular disease in competitive athletes. Circulation. 2015;132:e267-e72.

10. Drezner JA, O'Connor FG, Harmon KG, et al. AMSSM Position statement on cardiovascular preparticipation screening in athletes: current evidence, knowledge gaps, recommendations and future directions. Br J Sports Med. 2016;51:153-67.

11. Drezner J, Harmon K, Heistand J, et al. Effectiveness of emergency response planning for sudden cardiac arrest in United States high schools with automated external defibrillators. Circulation. 2009;120:518-25.

12. Sharma S, Drezner JA, Baggish A, et al. International recommendations for electrocardiographic interpretation in athletes. J Am Coll Cardiol. 2017;69:1057-75.

13. Sharma S, Drezner JA, Baggish A, et al. International recommendations for electrocardiographic interpretation in athletes. Eur Heart J. 2017; https://doi.org/10.1093/eurheartj/ehw631.

14. Drezner JA, Sharma S, Baggish A, et al. International recommendations for electrocardiographic interpretation in athletes. Br J Sports Med. 2017;51:704-31.

15. Panhuyzen-Goedkoop NM, Hoogsteen J. Pre-participation cardiovascular screening in young athletes in the Netherlands. Neth Heart J. 2005;13:335-7.

16. Corrado D, Pelliccia A, Heidbuchel H, et al. Recommendations for interpretation of 12-lead electrocardiogram in the athlete. Eur Heart J. 2010;31:243-59.
17. Pelliccia A, Fagard R, Bjornstad HH, et al. Recommendations for competitive sports participation in athletes with $\mathrm{CV}$ disease. Eur Heart J. 2005;26:1422-45.

18. Heidbuchel H, Papadakis M, Panhuyzen-Goedkoop N, et al. Position paper: proposal for a core curriculum for a European sports cardiology qualification. Eur J Prev Cardiol. 2013;20:889-903.

19. Rawlins J, Carre F, Kervio G, et al. Ethnic differences in physiological cardiac adaptation to intense physical exercise in highly trained female athletes. Circulation. 2010;121:1078-85.

20. Sheikh N, Papadakis M, Ghani S, et al. Comparison of electrocardiographic criteria for the detection of cardiac abnormalities in elite black and white athletes. Circulation. 2014;129:1637-49.

21. Riding NR, Sheikh N, Adamuz C, et al. Comparison of three current sets of electrocardiographic interpretation criteria for use in screening athletes. Heart. 2014;101:384-90.

22. Malhotra A, Walker M, Dhutia H, et al. Pre-participation screening in adolescent athletes: a comparison of European Society of Cardiology, Seattle and Refined ECG criteria: which is best? Heart. 2015;101:A45.

23. Drezner JA, Ackerman MJ, Anderson J, et al. Electrocardiographic interpretation in athletes: the Seattle criteria. Br J Sports Med. 2013;47:122-4.

24. Drezner JA, Fischbach P, Froelicher V, et al. Normal electrocardiographic findings: recognizing physiological adaptations in athletes. Br J Sports Med. 2013;47:125-36.

25. Drezner JA, Ashley E, Baggish AL, et al. Abnormal electrocardiographic findings in athletes: recognizing changes suggestive of cardiomyopathy. Br J Sports Med. 2013;47:137-52.

26. Sheikh N, Papadakis M, Carre F, et al. Cardiac adaptation to exercise in adolescent athletes of African ethnicity: an emergent elite athletic population. Br J Sports Med. 2013;47:585-92.

27. Duhtia H, Malhotra A, Gabus V, et al. Cost implications of using different ECG criteria for screening young athletes in the United Kingdom. J Am Coll Cardiol. 2016;16:702-11.

28. Panhuyzen-Goedkoop NM, Smeets JLRM. Legal responsibilities of physicians when making participation decisions in athletes with cardiac disorders: do guidelines provide a solid legal footing? $\mathrm{Br} \mathrm{J}$ Sports Med. 2014;48:1193-5.

29. Mitten MJ, Zipes DP, Maron BJ, et al. Eligibility and disqualification recommendations for competitive athletes with cardiovascular abnormalities: task force 15: legal aspects of medical eligibility and disqualification recommendations. Circulation. 2015;132:e346-e9.

30. Weiner RB, Hutter AM, Wang F, et al. Performance of the 2010 European Society of Cardiology criteria for ECG interpretation in the athlete. Heart. 2011;97:1573-7. 